\title{
Environmental optimization of tree-shaped water distribution networks
}

\author{
P. Bieupoude ${ }^{1}$, Y. Azoumah ${ }^{1} \&$ P. Neveu ${ }^{2}$ \\ ${ }^{I}$ International Institute for Water and Environmental Engineering (2iE) \\ Ouagadougou, Burkina Faso \\ ${ }^{2}$ Laboratoire PROcédés, Matériaux et Energie Solaire (PROMES), \\ Université de Perpignan Via Domitia, Perpignan, France
}

\begin{abstract}
In this paper two optimal tree-shaped networks for drinking water and irrigation are constructed by using the constructal approach coupled with the exergy destruction minimization method. It is shown that in the construction of treeshaped network for water distribution, the method of exergy destruction minimization is equivalent to minimizing mechanical irreversibility under a water quality constraint. For both energy consumption and the fluid quality degradation appearing in the network, this study offers new interesting routes for optimizing the system either by the exergy destruction minimization (in that case, both irreversible processes are taken into account in the optimization procedure) or by minimizing one of the two irreversible processes, the other being taken into account as optimization constraint. This paper shows that, for the improvement of performances it is important to focus on the design of an efficient distribution network rather than enhancing only the transport properties.

Keywords: water distribution network, constructal theory, exergy, optimization drinking water, irrigation.
\end{abstract}

\section{Introduction}

Several areas of engineering involve tree-shaped networks: electronic components cooling Bejan [1], heat and mass transfer in a solid-gas reactive porous media Azoumah et al. [2] or most generally flow structures [3, 4].

The optimal implementation of these flow systems depends greatly on their irreversibilities minimization. For the specific case of water piping systems two 
important phenomena are in competition: head losses leading to energy consumption and chemical losses causing water quality degradation $[5,6]$ showing that there is a trade-off to be found. This is the concern of this paper which uses concepts of Thermodynamics of irreversible process Prirogine [7] to describe the flow. Furthermore, Constructal design is performed and results are later analyzed from irrigation and drinking water constraints points of view.

\section{Thermodynamic analysis of a pressurized flow}

\subsection{Description of the system}

We learn from many applied hydraulics literature that head losses in a pipe (fig. 1) due to mechanical resistances is defined by eqn.(1) [8-10], where $a$ is a constant depending on the pipe material, $Q$ is the volumetric flow rate, $L$ the pipe length and $D$, its diameter. It is also known that the kinetic of chemical reaction in water pipe can be considered to be a first order kinetic close to water treatments reagents like chlorine residual which is modeled by a first order kinetic Rossman et al. [11]. From this, linking the residence time to the flow rate and the diameter, the variation of the concentration becomes eqn. (2).

$$
\begin{gathered}
\Delta H=a \frac{Q^{n}}{D^{m}} L \\
\Delta C=C_{0}\left(1-e^{-k \frac{\pi D^{2} L}{4 Q}}\right)
\end{gathered}
$$

where $C_{0}$ is the input concentration and $\left(\mathrm{t}=\pi \mathrm{D}^{2} \mathrm{~L} /(4 \mathrm{Q})\right)$ is the residence time. The analysis of the evolution of mechanical and chemical phenomena in the pipe shows that for a given diameter and flow rate, both $\Delta \mathrm{H}$ and $\Delta \mathrm{C}$ increase with the pipe length while they are antagonist over the diameter or over the flow rate $\mathrm{Q}$. Therefore, one can conclude that the pipe length does not affect irreversibility in the pipe in different ways while the diameter and the flow rate do.

\section{Input 1: Flow rate: $\mathrm{Q}$ Output 2:}

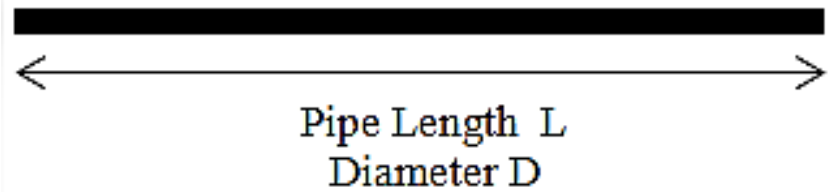

Figure 1: A given pipe in a pipes network.

The antagonist evolution of mechanical and chemical phenomena shows that there is a trade-off between chemical and mechanical phenomena to be determined (Fig. 2). But these two phenomena are not comparable in sense that mechanical irreversibilities are physical and chemical ones deals with quality. Setting thermodynamic balances as exposed in exergy destruction or entropy 


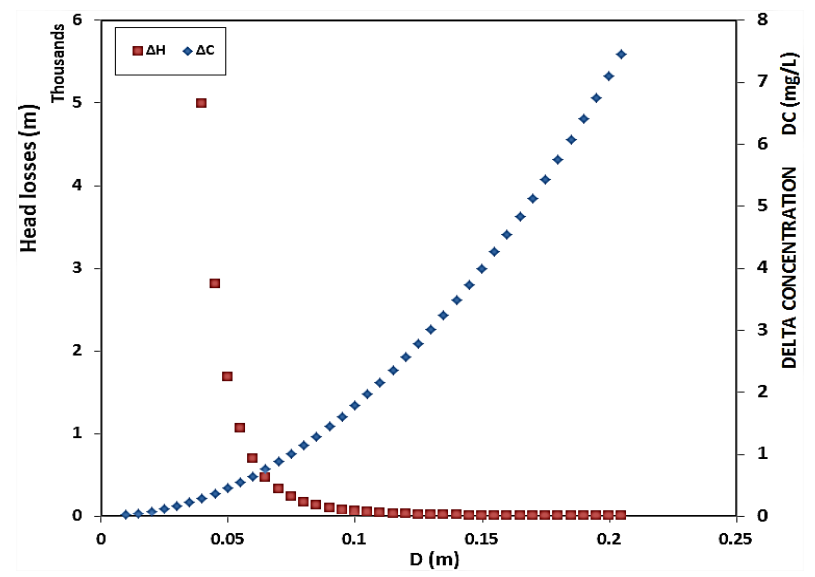

Figure 2: $\quad$ Head losses and concentration decay over the diameter.

generation papers $[2,7]$ can enable to account the contribution of all the phenomena occurring in the system.

\subsection{Thermodynamic balances of the flow}

\subsubsection{Assumptions and transient balances: mass, enthalpy and exergy}

Consider at a very simple scale the model of reaction occurring in the flow to be described as follow:

$$
A+B \rightarrow C+D
$$

$\mathrm{A}$ and $\mathrm{B}$ are reagents while $\mathrm{C}$ and $\mathrm{D}$ are the products of the reaction. With the assumption of ideal solution:

$$
\begin{aligned}
& \frac{d N_{H 2 O}}{d t}=\dot{n}_{1} \cdot\left(1-y_{A 1}-y_{B 1}-y_{C 1}-y_{D 1}\right)-\dot{n}_{2} \cdot\left(1-y_{A 2}-y_{B 2}-y_{C 2}-y_{D 2}\right) \\
& \frac{d E}{d t}=\dot{n}_{1} \cdot h_{1}-\dot{n}_{2} \cdot h_{2}-\dot{q}_{0} \\
& \frac{d\left(E-T_{0} S\right)}{d t}=\dot{n}_{1} \cdot e x_{1}-\dot{n}_{2} \cdot e x_{2}-\dot{q}_{0}(0)-e \dot{x} d
\end{aligned}
$$

where $\dot{\mathrm{n}}_{\mathrm{i}}$ is the molar flow rate $\left(\mathrm{mol} \mathrm{s}{ }^{-1}\right) ; y_{i}$ is the molar fraction of the $i^{\text {th }}$ constituent; $h_{i}$ is the total molar enthalpy of the solution $(\mathrm{J} / \mathrm{mole}) ; e x_{i}$ is the molar exergy of the solution (W/mole); $\dot{q}_{0}$ is the thermal power exchanged with surrounding at $\mathrm{T}_{0}$ temperature $(\mathrm{W}) ; k: 1$ : the input, 2 : the output. Assuming steady state and very weak concentrations of reactants and products $\left(\mathrm{y}_{\mathrm{i}}<<1\right)$, eqn. (4), results in 


$$
\begin{aligned}
& \dot{n}_{1} \approx \dot{n}_{2} \\
& \dot{q}_{0}=\dot{n}_{1} \cdot\left(h_{1}-h_{2}\right) \\
& e \dot{x}_{d}=\dot{n}_{1} \cdot\left(e x_{1}-e x_{2}\right)
\end{aligned}
$$

\subsubsection{Thermodynamic analysis of the flowing fluid}

2.2.2.1 Molar enthalpy of the solution The total molar enthalpy of the fluid is expressed by eqn.(6). $h(T, p, y)$ is the molar enthalpy of the solution $(\mathrm{J} / \mathrm{mole})$; $\mathrm{M}$ is the molar mass of the solution, $\mathrm{V}$ is water velocity in the pipe $\left(\mathrm{m} . \mathrm{s}^{-1}\right)$; $\mathrm{g}$ is the gravitational acceleration $\left(\mathrm{m} / \mathrm{s}^{2}\right) ; \mathrm{z}$ is the altitude $(\mathrm{m})$. The total molar enthalpy of this solution (under the consideration of $y_{\mathrm{i}}<<1$ ) is given by eqn. (7).

$$
\begin{gathered}
h=h(T, \quad p, y)+M . V=/ 2+g z \\
h(T, p, y) \approx \cdot h_{H 2 O}^{0}(T, p)+c_{H 2 O} \cdot\left(T-T^{0}\right)+v_{H 2 O} \cdot\left(p-p^{0}\right)
\end{gathered}
$$

where $\mathrm{c}_{\mathrm{H} 2 \mathrm{O}}$ and $\mathrm{v}_{\mathrm{H} 2 \mathrm{O}}$ are respectively the molar thermal capacity and the molar volume of the water. 0 represents the reference state.

2.2.3 Molar entropy Molar entropy of an ideal solution can be expressed by:

$$
\begin{aligned}
s(T, p, y)= & \left(1-y_{A}-y_{B}-y_{C}-y_{D}\right) \cdot\left[s_{H 2 O}(T, p)-R \cdot \operatorname{Ln}\left(1-y_{A}-y_{B}-y_{C}-y_{D}\right)\right] \\
& +y_{A} \cdot\left[s_{A}(T, p)-R \cdot \operatorname{Ln}\left(y_{A}\right)\right]+y_{B} \cdot\left[s_{B}(T, p)-R \cdot \operatorname{Ln}\left(y_{B}\right)\right] \\
& +y_{D} \cdot\left[s_{D}(T, p)-R \cdot \operatorname{Ln}\left(y_{D}\right)+y_{C} \cdot\left[s_{C}(T, p)-R \cdot \operatorname{Ln}\left(y_{C}\right)\right]\right]
\end{aligned}
$$

where $\mathrm{S}_{\mathrm{H} 2 \mathrm{O}}(\mathrm{T}, \mathrm{p}), \mathrm{s}_{\mathrm{A}}(\mathrm{T}, \mathrm{p}), \mathrm{s}_{\mathrm{B}}(\mathrm{T}, \mathrm{p})$ et $\mathrm{s}_{\mathrm{Cl}}(\mathrm{T}, \mathrm{p})$ are specific molar enthalpies. With the earlier consideration $\left(\mathrm{y}_{\mathrm{i}}<<1\right)$ and the incompressibility of the fluid

$$
\begin{aligned}
s(T, p, y) \approx & \cdot S_{\text {нго }}^{o}(T, p)+c_{\text {нго }} \cdot \operatorname{Ln}\left(\frac{T}{T^{0}}\right) \\
& -R \cdot\left[\begin{array}{l}
\left(1-y_{A}-y_{B}-y_{C}-y_{D}\right) \operatorname{Ln}\left(1-y_{A}-y_{B}-y_{C}-y_{D}\right) \\
+y_{A} \operatorname{Ln}\left(y_{A}\right)+y_{B} \operatorname{Ln}\left(y_{B}\right)+y_{C} \operatorname{Ln}\left(y_{C}\right)+y_{D} \operatorname{Ln}\left(y_{D}\right)
\end{array}\right]
\end{aligned}
$$

2.2.2.3 Molar exergy The molar exergy of the solution can be deduced directly from eqns. (7), (8) and (9) as follows:

$$
\begin{aligned}
\operatorname{ex}(T, p, y, V, z) \approx & h_{\text {нго }}^{o}(T, p)-T^{0} \cdot s_{\text {нго }}^{o}(T, p) \\
& +c_{\text {нго }} \cdot\left(\left(T-T^{0}\right)-T^{0} \operatorname{Ln} \frac{T}{T^{0}}\right)+v_{\text {н2о }} \cdot\left(p-p^{0}\right) \\
& +M \cdot V^{2} / 2+g \cdot z \\
& +R \cdot T^{o} \cdot\left[\begin{array}{l}
\left(1-y_{A}-y_{B}-y_{C}-y_{D}\right) \operatorname{Ln}\left(1-y_{A}-y_{B}-y_{C}-y_{D}\right) \\
+y_{A} \operatorname{Ln}\left(y_{A}\right)+y_{B} \operatorname{Ln}\left(y_{B}\right)+y_{C} \operatorname{Ln}\left(y_{C}\right)+y_{D} \operatorname{Ln}\left(y_{D}\right)
\end{array}\right]
\end{aligned}
$$

2.2.2.4 Formula of the total destruction of the exergy Finally, the exergy destruction is obtained from previous equations as follows: 


$$
e \dot{x}_{d}=\dot{n}_{1} \cdot\left[\begin{array}{l}
c_{\text {H2O }} \cdot\left(T_{1}-T_{2}-T^{0} \operatorname{Ln} \frac{T_{1}}{T_{2}}\right)+v_{\text {H2O }} \cdot\left(p_{1}-p_{2}\right) \\
+M \cdot\left(V_{1}^{2}-V_{2}^{2}\right) / 2+g \cdot\left(z_{1}-z_{2}\right)-R \cdot T^{0} \cdot \operatorname{Ln}\left[\frac{Y_{2}}{Y_{1}}\right]
\end{array}\right]
$$

$\left(\mathrm{Y}_{2} / \mathrm{Y}_{1}\right)$ is the variation of terms with log, eqn. (10), from input 1 to output 2 . The variation of the water temperature is negligible from the input point to the output point $\left(T_{1}=T_{2}\right)$. The velocity is also supposed to be constant, $V_{1}=V_{2}$. Then the introduction of pressure drops equation (equal to head losses) given by [9] permit finally to have:

$$
e \dot{x}_{d}=\dot{n}_{1} \cdot\left(\operatorname{ag} \cdot M_{e} \cdot \frac{Q^{n}}{D^{m}} \cdot L-R \cdot T^{0} \cdot \operatorname{Ln}\left[\frac{Y_{2}}{Y_{1}}\right]\right)
$$

By this function chemical and mechanical irreversibilities have been assessed and quantified. Now, the concern of the coming section will be the analysis of this function.

\subsubsection{Total exergy destruction analysis}

In this section, a large interval of molar fraction is considered to analyze the evolution of the exergy destruction as shown in Fig. 3.

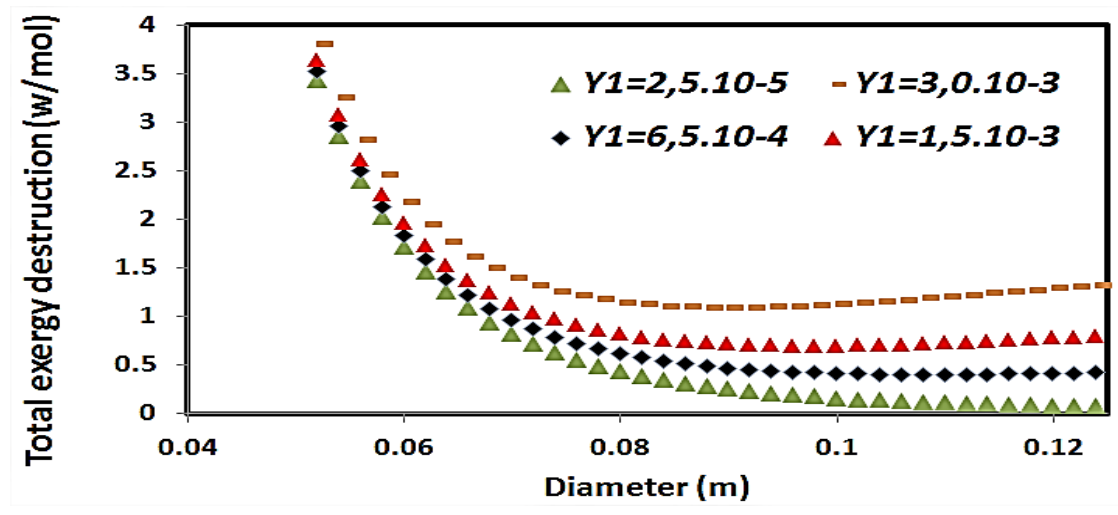

Figure 3: Total exergy destruction for different molar fraction over the diameter.

Two different situations are presented. On one hand, when the molar fraction is high, an optimal diameter that leads to the total exergy destruction minimization exists. On the other hand for low values of molar fraction ranging from $10^{-4}$ to $10^{-7}$, the diameter that minimizes the exergy destruction is found for very low flow velocities $(<0.4 \mathrm{~m} / \mathrm{s})$. This second situation is generally met in drinking water distribution area because of the WHO (World Health Organization) [12] regulation specifications that are applied to ensure water quality. Indeed, for drinking water, initially treated and put in distribution 
network, a good concentration of chemicals like chlorine residuals means a good quality of water. In fact, the quality agents like chlorine residuals, destroy pathogenic microorganisms which cause typhoid, hepatitis, cholera or bacillary dysentery Crittenden [13] but their concentrations decrease with the residence time until they become completely imperceptible at the end of the pipe Rodriguez et al. [14]. For instance, in USA, the Environmental Protection Agency, USEPA imposes chlorine residuals concentration closed to $0.2 \mathrm{mg} / \mathrm{l}$ at least in water distribution pipes after water treatment points EPA [15].

Though the above result seems to be original, interesting and more adapted for some chemical engineering fields (sanitation, depollution) [16-18], it cannot be applied to the field of drinking water network optimization since molar fractions of constituents in this field, are very low ranging around $10^{-6}$ due to the WHO regulation on chlorine residuals WHO [12]. Therefore, it seems more judicious and relevant to reduce the exergy destruction to its mechanical contribution which is the most important part from a thermodynamic point of view and consider the chemical contribution as a constraint. By this way, the problem is reduced to mechanical irreversibility minimization under water quality constraint.

\section{Construction of the water distribution networks}

The method of exergy destruction minimization, coupled with the constructal approach, is used to optimize and to construct two water distribution networks solving then the above formulated problem. The construction will be done from a basic elemental network to the overall network obtained after a certain level of construction.

\subsection{Constructal sequences: from 1 to higher-order constructs}

Consider a k-levels of pairing network for both two-branching (N2N) and fourbranching (N4N) configurations to cover a given area. For example $k=2$ and $k=4$ results in fig. 4 . The head losses and residence time in each system are given by:

$$
\Delta H=a \cdot \sum_{i=0}^{k} \frac{Q_{i}{ }^{n}}{D_{i}{ }^{m}} \cdot L_{i} \quad ; \quad t=\frac{\pi}{4} \sum_{i=0}^{k} L_{i} D_{i}{ }^{2} / Q_{i}
$$

By the method of Lagrange multipliers, the linear combination of these two relations leads to:

$$
L_{g}=a \cdot \sum_{i=0}^{k} \frac{Q_{i}^{n}}{D_{i}^{m}} \cdot L_{i}+\lambda\left(\frac{\pi}{4} \sum_{i=0}^{k} L_{i} D_{i}^{2} / Q_{i}-t\right)
$$

When we solve equations $\partial \mathrm{Lg} / \partial \mathrm{D}_{\mathrm{i}}=0$ and eliminate $\lambda$, we then obtain: 


$$
D_{o}=\sqrt{\frac{t Q_{0}}{L_{o}+\sum_{i=1}^{k} L_{i}\left(\prod_{i=1}^{i} N_{p}\right)^{\left(\frac{n+1}{m+2}-l\right)}}} ; \quad\left(\frac{D_{k}}{D_{k-l}}\right)=N_{p}^{\frac{n+1}{m+2}} ; \frac{D_{k}}{D_{o}}=N_{p}^{k \frac{n+1}{m+2}}
$$

These relations represent the optimal diameters distribution through the network. The minimal head needed of the network upstream is obtained by reevaluating the eqn. (13).

$$
H_{1}=H_{0}+a\left(L_{0}+\sum_{i=1}^{k} N_{p}^{\left(n-m \frac{n+1}{m+2}\right) i} L_{i}\right) J_{0}
$$
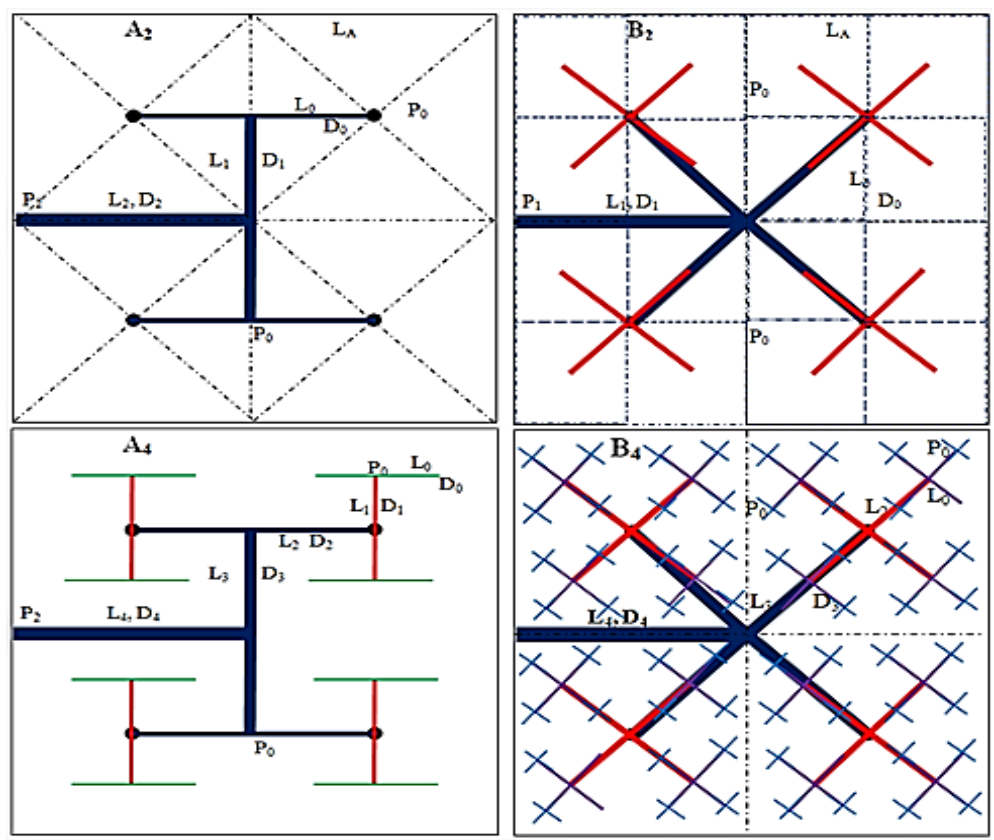

Figure 4: Configuration of constructs.

\section{Results and discussion}

For each construct, a law of optimal diameter distribution has been determined to minimize mechanical irreversibility which is linked to pumping power of the network. For all the constructs, the optimal solutions depend strongly on both the number of pairings $N_{p}$ at nodes and the number of pairing levels $k$. Velocities analysis showed that for both configurations, from the first construct to higherorder constructs, the velocities intervals increase as shown in Fig. 5. Especially, velocities intervals vary faster for $\mathrm{N} 4 \mathrm{~N}$ than for $\mathrm{N} 2 \mathrm{~N}$ meaning that velocities 
distribution is more regular for $\mathrm{N} 2 \mathrm{~N}$ than for N4N. For lower-order constructs of $\mathrm{N} 4 \mathrm{~N}$, the maximal velocities are lower than the maximal velocities of $\mathrm{N} 2 \mathrm{~N}$. But they become superior to N2N's for higher-order constructs. On contrary, minimal velocities have an opposite evolution. For lower construction scales, $\mathrm{N} 2 \mathrm{~N}$ have lower velocities, but this is not the case for higher-order constructs.

This velocities distribution results in the average velocities evolution shown in Fig. 6. The average velocities of the N2N are inferior to N4N's for lowerorder constructs.

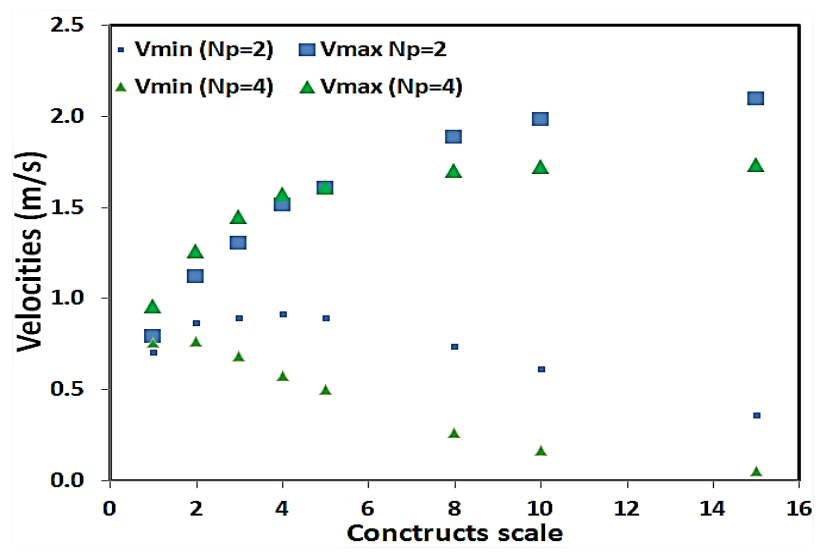

Figure 5: $\quad$ Max and min velocities in N2N and N4N networks.

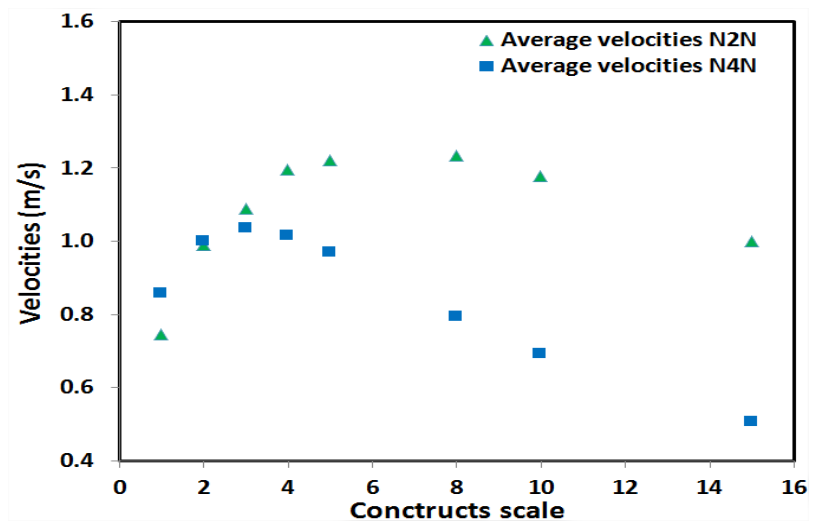

Figure 6: Average velocities through N2N and N4N networks.

These average velocities distribution permit to understand the evolution of the pumping power and the average unit pressure drops through the networks as shown in Fig. 7. 

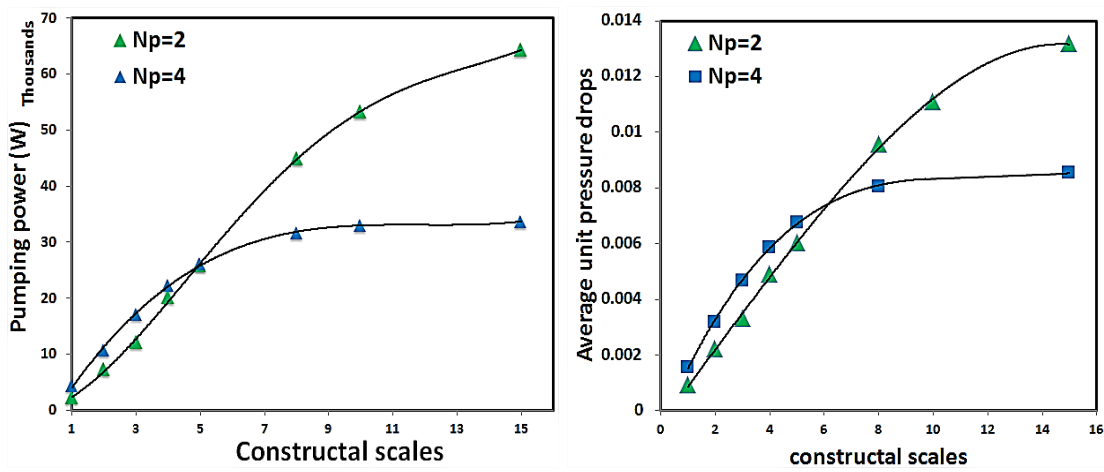

Figure 7: $\quad$ Pumping power and unit pressure over constructs scales.

\section{Domains of application}

Fig. 7 shows that the unit pressure drops are inferior for $\mathrm{N} 4 \mathrm{~N}$ to $\mathrm{N} 2 \mathrm{~N}$ when higher-order constructs are considered. Besides, average velocities in N4N are inferior to N2Ns ones and the number of irrigated points is the highest; meaning that N4N can irrigate high concentration points well for a given surface. These criteria of low velocities and a large number of points to be served are precisely met in the domains of irrigation in agriculture, according to literature on irrigation technique [19-21]. For these reasons, the N4N networks considered in higher-order constructs seems to be suitable for irrigation. But for drinking water systems, the water quality evolution is well known according to many authors [22-24] to be degradable with the residence time. That is why it is pertinent from an environmental protection point of view (good control of water quality) to consider the $\mathrm{N} 2 \mathrm{~N}$ networks as the most adapted to drinking water distribution systems for their good flow velocities distribution through the networks.

\section{Conclusion}

This paper offers a new interesting route of optimizing tree-shaped water distribution networks through exergy destruction or pumping power minimization. Water demands and topography variation or minor losses have not been considered here but for further investigations they could be included to be close to a realistic water distribution network.

This study showed that for the improvement of performances, it is important to focus on the design of an efficient distribution network rather than enhancing only the transport properties. Note finally that the focus on the quality preservation in flow systems is a pertinent approach in engineering and environmental protection in such drinking water distribution systems. 


\section{References}

[1] Bejan, A., Constructal-theory network of conducting paths for cooling a heat generating volume, International Journal of Heat Mass Transfer, 40(4), pp.799-816,1997.

[2] Azoumah, Y., Mazet, N. \& Neveu, P. Constructal network for heat and mass transfer in a solid-gas reactive porous medium, International Journal of Heat Mass Transfer, 47(14-16), pp. 2961-2970, 2004.

[3] Bejan, A. \& Lorente, S., Constructal tree-shaped flow structures, Applied Thermal Engineering, 27(4), pp. 755-761, 2007.

[4] Tondeur, D. \& Luo, L., Design and scaling laws of ramified fluid distributors by the constructal approach, Chemical Engineering Science, 59(8-9), pp. 1799-1813, 2004.

[5] Izquierdo, J., Montalvo, T., Pérez, R. \& Herrera, M., Sensitivity analysis to assess the relative importance of pipes in water distribution networks, Mathematical and Computer Modelling, 48(1-2), pp. 268-278, 2008.

[6] Kerneïs, A., Nakache, F., Deguin, A. \& Feinberg, M., The effects of water residence time on the biological quality in a distribution network, Water research, 29(7), pp. 1719-1727, 1995.

[7] Prirogine, I., Introduction to Thermodynamics of Irreversible Process, Wiley, New York, 1962.

[8] Calier, M., Hydraulique Générale et appliquée, Eyrolles, Paris, 1972.

[9] Lahiouel, Y., Haddad, A.K. \& Chaoui, K., Evaluation of head losses in fluid transportation networks, Sciences \& Technologie, 23, pp. 89-94, 2005.

[10] Walski, T., Chase, D. \& Savic, D., Water distribution modeling, Heated method, Heasted press, USA, 2001.

[11] Rossman, L.A., Clark, R.M. \& Grayman, W.M., Modeling chlorine residual in drinking -water distribution system, Journal of Environmental Engineering, 120 (4), pp. 803-820, 1994.

[12] World Health Organization, Guidelines for Drinking-Water Quality, 2, $2^{\text {nd }}$ Edition, Health Criteria and Other Supporting Information. WHO, Mastercom/Wiener Verlag, 1996.

[13] Crittenden, J., Trussell, R., Hand, D., Howe, K. \& Tchobanoglous, Water Treatment, Principles and Design, MWH, $2^{\text {nd }}$ Edition, John Wiley \& Sons, New York, 2005.

[14] Rodriguez, M., Milot, J., Sérodes, J. \& Pacaud, A. Estimation of benchscale chlorine decay in drinking water using nth order kinetic and back propagation neural network models, Water Quality Research, 37 (3), pp. 613-635, 2001.

[15] Environmental Protection Agency (EPA), National primary drinking water regulations: Disinfectants and disinfection by products rule; Final rule. Federal Register, 63(241), pp. 69390-69476, 1998.

[16] Torrijos, M. \& Moletta, R. Winery wastewater depollution by sequencing batch reactor, Water Science and Technology, 35, pp. 249-257.

[17] Azrague, K., Aimar, P., Benoit-Marquié, F. \& M.T. Maurette. A new combination of a membrane and a photocatalytic reactor for the depollution 
of turbid water, Applied Catalysis B: Environmental, 72 (3-4), pp. 197-204, 2007.

[18] A. Montangero, and al. Optimising water and phosphorus management in the urban environmental sanitation system of Hanoi, Vietnam, Science of The Total Environment, 384(1-3), pp. 55-66, 2007.

[19] Zella, L. \& Kettab, A., Optimisation d'un réseau de microirrigation = Optimising a microirrigation network, sécheresse, 14(3), pp. 189-194, 2003.

[20] Calejo, M., Lamaddalena, N., Teixeira, J. \& Pereira, L., Performance analysis of pressurized irrigation systems operating on-demand using flowdriven simulation models, Agricultural Water Management, 95(2), pp. 154162, 2008.

[21] De La Bouëre, J., Micro-irrigation, bases et principes DVD réf MA2602, Lavoisier 2000-2010, 2006.

[22] Rossman, L. \& Boulos, P., Numerical methods for modeling water quality in distribution systems: a comparison, Journal of Water Resources Planning and Management, 122(2), pp. 137-146, 1996.

[23] Shang, F., Uber, J. \& L. Rossman, Modeling reaction and transport of multiple species in water distribution systems, Environmental Science \& Technology, 42(3), pp. 808-814, 2008.

[24] Clarck, R., Rossman, L. \& Wymer, L., Modeling distribution system water quality: regulatory implications, Journal of water resources planning and management, 121(6), pp. 423-428, 1995.

[25] Tondeur, D., Fan, Y. \& Luo, L., Constructal optimization of arborescent structures with flow singularities, Chemical Engineering Science, 64(18), pp. $3968-3982,2009$. 